Sammlung Metzler

Band 36 


\author{
Joachim Bumke
}

\title{
Wolfram von Eschenbach
}

7., völlig neu bearbeitete Auflage

Verlag J.B. Metzler

Stuttgart - Weimar 
Die Deutsche Bibliothek - CIP-Einheitsaufnahme

Bumke, Joachim:

Wolfram von Eschenbach / Joachim Bumke.

- 7., völlig neu bearb. Aufl.

- Stuttgart ; Weimar : Metzler, 1997

(Sammlung Metzler ; Bd. 36)

ISBN 978-3-476-17036-1

ISBN 978-3-476-17036-1

ISBN 978-3-476-04150-0 (eBook)

DOI $10.1007 / 978-3-476-04150-0$

ISSN 0058-3667

SM 36

Dieses Werk einschließlich aller seiner Teile ist urheberrechtlich geschützt. Jede Verwertung außerhalb der engen Grenzen des Urheberrechtsgesetzes ist ohne Zustimmung des Verlages unzulässig und strafbar. Das gilt insbesondere für Vervielfältigungen, Übersetzungen, Mikroverfilmungen und die Einspeicherung und Verarbeitung in elektronischen Systemen.

(C) 1997 Springer-Verlag GmbH Deutschland Ursprünglich erschienen bei J.B. Metzlersche Verlagsbuchhandlung und Carl Ernst Poeschel Verlag GmbH in Stuttgart 1997 


\section{Vorwort zur 7. Auflage}

Für die 7. Auflage wurde der Text in allen Teilen überarbeitet. Größere Ergänzungen waren in den Abschnitten "Interpretationsprobleme" sowohl im ,Parzivalı-Kapitel als auch im ,Willehalm - Kapitel und außerdem im Abschnitt "Entstehung, Überlieferung, Wirkungsgeschichte" des ,Parzival--Kapitels notwendig. Der Abschnitt "Der Erzähler am Werk" im ,Parzival-Kapitel ist neu dazugekommen. Die Literaturangaben wurden durch die seit der 6 . Auflage erschienenen Titel ergänzt. Andere geplante Ergänzungen konnten noch nicht verwirklicht werden.

Die Erweiterung des Umfangs mußte aus verlagstechnischen Gründen durch Kürzungen wenigstens teilweise wettgemacht werden. Trotz schwerwiegender Bedenken habe ich mich entschlossen, die Literaturangaben zu kürzen. Die Kürzungen betreffen in erster Linie die Forschung bis in die Mitte der 80er Jahre (die in den früheren Auflagen dieses Bändchens nachgeschlagen werden kann). Zitiert werden nur noch die Arbeiten, die den Forschungsstand bis heute prägen. Generell liegt der Akzent auf der Forschung der letzten zehn Jahre. Aber auch für die neuere Forschung ist Vollständigkeit nicht angestrebt. Ich verweise auf die umfangreiche und sorgfältige Dokumentation der neueren Forschung in der Wolfram-Bibliographie von Renate Decke-Cornill, die seit 1988 in den WolframStudien (ab Band 10) erscheint.

Köln, Nov. 1996

J.B. 


\section{Inhalt}

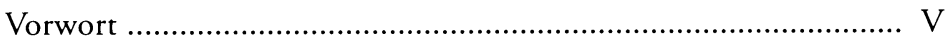

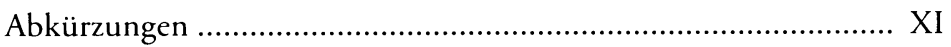

I. Der Dichter in seiner Zeit ..................................................... 1

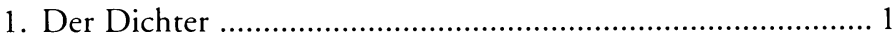

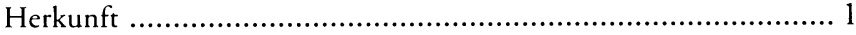

Standesverhältnisse ................................................................ 4

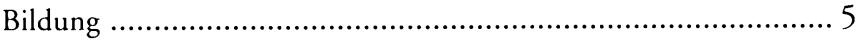

2. Gönner und Publikum …………………………………..... 8

Landgraf Hermann von Thüringen ............................................ 9

Der fränkisch-bayerische Gönnerkreis ........................................ 11

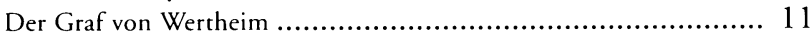

Burg Wildenberg ........................................................ 11

Der Anger von Abenberg ............................................... 12

Trüdingen und Dollnstein .............................................. 13

Die Markgräfin von Haidstein ......................................... 13

Heinrich von Rispach ................................................. 14

Ein wîp .................................................................... 14

3. Literarische Beziehungen .............................................. 14

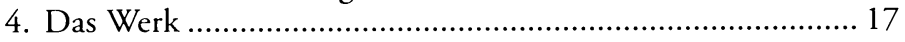

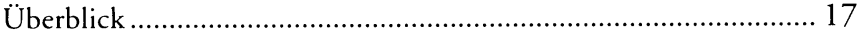

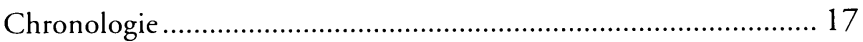

Sprache und Stil ...................................................................... 19

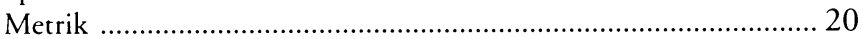

5. Wirkungsgeschichte .................................................... 21

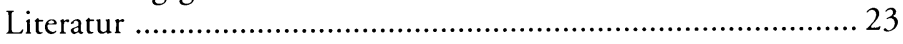

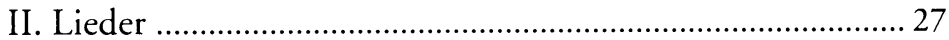

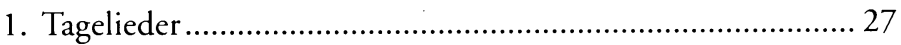

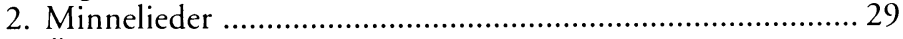

3. Überlieferung und Wirkungsgeschichte .................................. 30

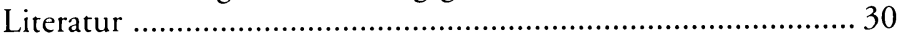




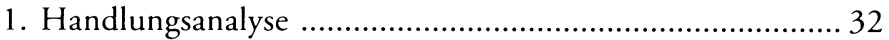

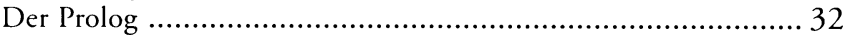

Die Geschichten von Gahmuret (Buch I-II) ........................... 34

Die erste Parzival-Partie (Buch III- VI) .................................. 42

Die erste Gawan-Partie (Buch VII-VIII) .................................. 62

Parzival bei Trevrizent (Buch IX) ...........................................69 69

Die zweite Gawan-Partie (Buch X-XIV) ................................ 76

Der Abschluß der Parzivalhandlung (Buch XV-XVI) ................. 94

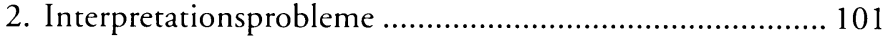

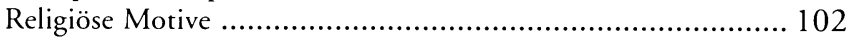

Liebe und Ehe. Frauengestalten und Geschlechterverhältnis ..... 113

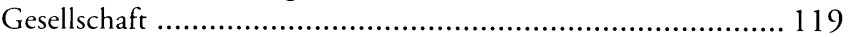

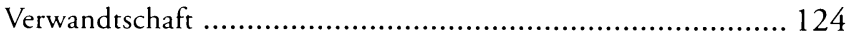

3. Der Erzähler am Werk .............................................. 128

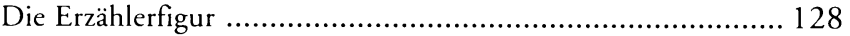

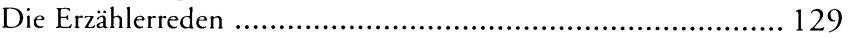

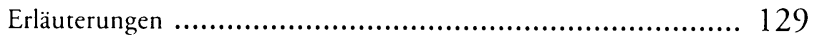

Sentenzen und Sprichwörter ....................................... 129

Minne-Exkurse ............................................................ 130

Zeitgeschichtliche Anspielungen ..................................... 130

Literarische Anspielungen ............................................. 131

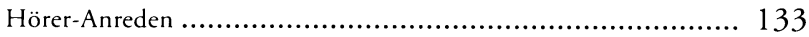

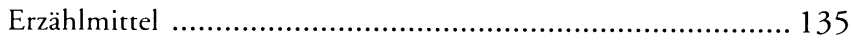

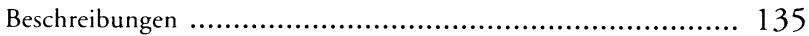

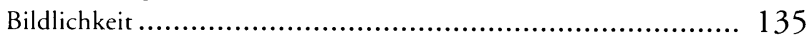

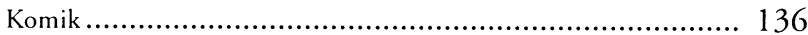

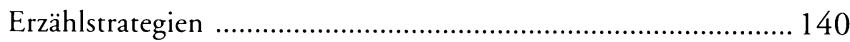

Zeit und Raum ....................................................... 140

Verknüpfungen ........................................................... 143

4. Komposition: Bücher und Dreißiger .............................. 151

5. Der Stoff und seine Bearbeitung .................................... 154

Die Parzival-Gral-Sage ...................................................... 154

Wolframs Hauptquelle: Der ,Conte du Graak von

Chrétien de Troyes ............................................................. 158

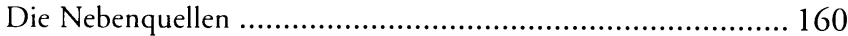

Keltische Quellen ....................................................... 160

Französische Quellen ................................................. 160

Orientalische Quellen .................................................... 162

Lateinische Quellen ....................................................... 163

Deutsche Quellen ........................................................ 164

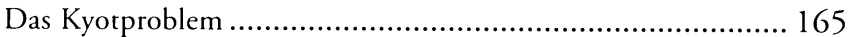

6. Entstehung, Überlieferung, Wirkungsgeschichte............... 167 


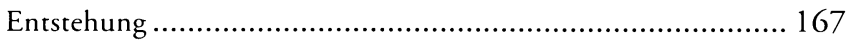

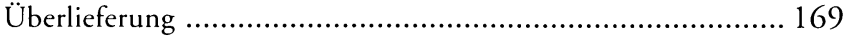

Überlieferungsfakten .............................................. 169

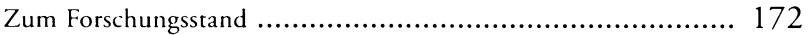

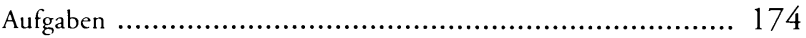

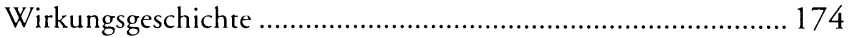

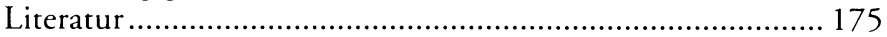

IV. Willehalm ................................................................ 190

1. Handlungsanalyse ...................................................... 190

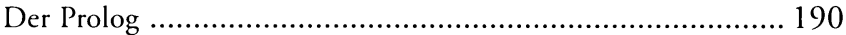

Die erste Schlacht auf Alischanz (Buch I-II) .......................... 192

Zwischen den Schlachten (Buch III-VI) ............................... 197

Die zweite Schlacht auf Alischanz (Buch VII-IX) ................... 207

2. Interpretationsprobleme .............................................. 214

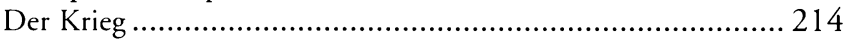

Der Kreuzzugsgedanke ................................................ 215

Der Reichsgedanke ..................................................... 216

Der Krieg der Worte ................................................... 218

Das Heidenbild ...................................................... 218

Der religiöse Friedensgedanke ......................................... 221

Die menschlichen Bindungen ........................................... 221

Die gesellschaftlichen Bindungen ...................................... 222

Die zwischengeschlechtlichen Bindungen: Liebe und Ehe .......... 224

Die familiären Bindungen: Die Bedeutung der Verwandtschaft .... 226

Die religiösen Bindungen ................................................ 229

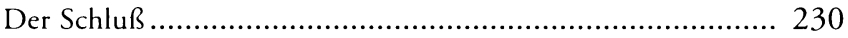

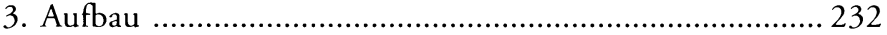

4. Der Stoff und seine Bearbeitung .................................. 233

Historische Grundlagen .................................................... 233

Der französische Epenzyklus um Guillaume d'Orange .............. 233

Wolframs Vorlage: Aliscans ‘............................................... 234

Die Bearbeitung der Quelle ............................................. 235

5. Überlieferung und Wirkungsgeschichte ......................... 236

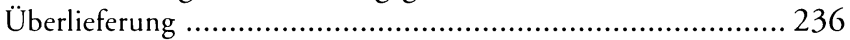

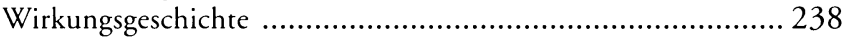

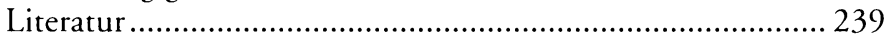

V. Titurel ........................................................................... 246

1. Handlungsanalyse ................................................. 246

Das erste Fragment ....................................................... 246 


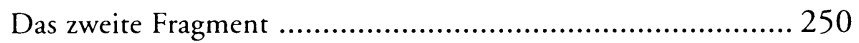

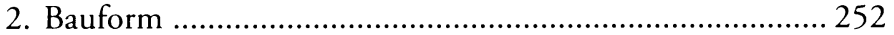

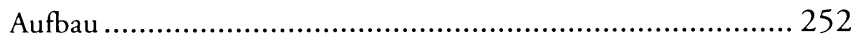

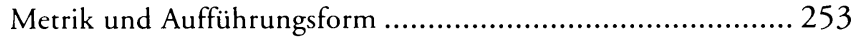

3. Der Stoff und seine Bearbeitung ................................. 254

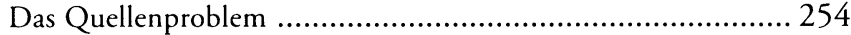

Das Verhältnis zum ,Parzival ............................................... 255

4. Überlieferung und Wirkungsgeschichte ........................ 256

Überlieferung ............................................................... 256

Wirkungsgeschichte ............................................... 256

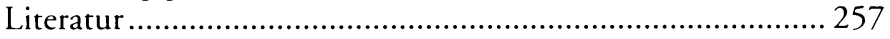

Register .................................................................. 260

Register zur Forschung .............................................. 260

Sachregister ............................................................. 265

Angaben zum Autor .............................................. 274 


\section{Verzeichnis der Abkürzungen}

$\mathrm{ABäG}$

AfdA

AG

AKG

Arch.

ATB

Beitr.

CCM

CG

DU

DVjs.

Et. germ.

Euph.

FMLS

FrSt.

FS

GLL

GQ

GR

GRM

IASL

Jb.

$\mathrm{Hs}(\mathrm{s})$.

JEGP

Lit. Jb.

MA

MF

MLN

MLQ

MLR

Neoph.

Neuph. Mitt.

$\mathrm{PhQ}$
Amsterdamer Beiträge zur älteren Germanistik Anzeiger für deutsches Altertum

Acta Germanica

Archiv für Kulturgeschichte

Archiv für das Studium der neueren Sprachen

Altdeutsche Textbibliothek

Beiträge zur Geschichte der deutschen Sprache und Literatur

Cahiers de civilisation médiévale

Colloquia Germanica

Der Deutschunterricht

Deutsche Vierteljahrschrift für Literaturwissenschaft und Geistesgeschichte

Études germaniques

Euphorion

Forum for Modern Language Studies

Frühmittelalterliche Studien

Festschrift

German Life and Letters

German Quarterly

Germanic Review

Germanisch-Romanische Monatsschrift

Internationales Archiv für Sozialgeschichte der deutschen Literatur

Jahrbuch

Handschrift(en)

Journal of English and Germanic Philology

Literaturwissenschaftliches Jahrbuch

der Görres-Gesellschaft

Medium Aevum

Minnesangs Frühling

Modern Language Notes

Modern Language Quarterly

Modern Language Review

Neophilologus

Neuphilologische Mitteilungen

Philological Quarterly 
PMLA Publications of the Modern Language Association

RF Romanische Forschungen

SB Sitzungsberichte

St. med. Studi medievali

Stud. neoph. Studia neophilologica

VL

Die deutsche Literatur des Mittelalters,

Verfasserlexikon $\left({ }^{2} \mathrm{VL}=2\right.$. Aufl. $)$

WmhM Würzburger medizinhistorische Mitteilungen

WvE, Wd'E Wolfram von Eschenbach

WW

Wirkendes Wort

ZfdA

Zeitschrift für deutsches Altertum

ZfdPh.

Zeitschrift für deutsche Philologie

ZfdSp.

Zeitschrift für deutsche Sprache

ZfrPh.

Zeitschrift für romanische Philologie

Der > Parzival< wird nach der Ausgabe von E. Nellmann (vgl. S. 175) zitiert; der $>$ Willehalm< nach der Ausgabe von J. Heinzle (vgl. S. 239); der Titurel nach der Ausgabe von K. Lachmann (vgl. S. 257); die Lieder nach der Ausgabe von P. Wapnewski (vgl. S. 30). 